\title{
Relação entre a pressão muscular perineal no puerpério e o tipo de parto
}

\author{
Relationship between perineal muscular force in the puerperal period and the type of delivery
}

Silmara Menta ${ }^{1}$, Janine Schirmer ${ }^{2}$

\section{Resumo}

Objetivo: relacionar o tipo de parto e as características do períneo com valores da pressão muscular perineal (PMP) mensurada em primíparas nas posições deitada e sentada com a musculatura perineal em repouso e em contração máxima. Métodos: estudo quantitativo do tipo transversal, realizado em maternidade conveniada ao Sistema Único de Saúde (SUS) do município de São Paulo. A casuística, obtida por conveniência, foi de 95 primíparas de termo. A avaliação ocorreu entre o $40^{\circ}$ e o $45^{\circ}$ dia. Realizaram-se entrevista, exame físico e mensuração da PMP por meio do perineômetro de Kegel. A mensuração foi realizada nas posições deitada e sentada, com a musculatura em repouso e em contração máxima, sendo considerada a média de três aferições para cada posição e estado muscular. Resultados: $76,8 \%$ (73) das primíparas tiveram parto vaginal e $23,2 \%$ (22) cesárea. No pós-parto vaginal, observou-se períneo íntegro em 18,9\% (18), com rotura perineal em $24,2 \%$ (23) e com episiotomia em 33,7\% (32). Os valores obtidos da PMP foram em: posição deitada/musculatura em repouso, 18,9 $\mathrm{mmHg}$; deitada/musculatura em contração máxima, 30,7 $\mathrm{mmHg}$; sentada/musculatura em repouso, 34,5 mmHg; sentada/musculatura em contração máxima, $46,5 \mathrm{mmHg}$. Conclusão: não houve associação entre o tipo de parto e as condições perineais e a pressão muscular perineal.

PALAVRAS-CHAVE: Parto; Parto normal/enfermagem; Assoalho pélvico; Períneo

\section{Abstract}

Purpose: to determine the values of perineal muscular force (PMF) in the lying and seated positions and to identify the values of PMF between first pregnancy, according to type and the characteristics of the vaginal delivery and cesarean section. Methods: study of the transversal type, performed in a maternity of Brazilian Public the Health System (SUS) in the city of São Paulo. The sample consisted of 95 primiparae at term. Evaluation occurred between the $40^{\text {th }}$ and 45 th, day with an interview, physical examination and measurement of PMF using a perineometer of the Kegel type. The measurement was carried out in the lying and seated positions, muscular status (at rest and in maximum contraction), and the average of three measures for each position and muscular state were considered. Results: $76.8 \%$ (73) of the women had vaginal delivery and $23.2 \%$ (22) cesarean section. After vaginal delivery, intact perineum in $18.9 \%$, (18), perineal rupture in $24.2 \%$ (23), and episiotomy in 33.7\% (32) were observed. Obtained values of the PMF were: lying position muscular rest $18.9 \mathrm{mmHg}$, lying position maximum contraction: $30,7 \mathrm{mmHg}$, seated position muscular rest: $34.5 \mathrm{mmHg}$, seated positions maximum muscular contraction: $46.5 \mathrm{mmHg}$. Conclusion: there was association between the type and the characteristics of the delivery and PMF.

KEYWORDS: Parturition; Natural childbirth/nursing; Pelvic floor; Perineum

\footnotetext{
1 Pós-graduanda de Programa de Pós-Graduação em Enfermagem do Departamento de Enfermagem da Universidade Federal de São Paulo UNIFESP - São Paulo (SP), Brasil.

2 Professora Associada da Disciplina Enfermagem Obstétrica do Departamento de Enfermagem da Universidade Federal de São Paulo UNIFESP - São Paulo (SP), Brasil; Consultora da Área Técnica Saúde da Mulher do Ministério da Saúde - Brasília (DF), Brasil. Correspondência: Janine Schirmer

Rua Leandro Dupre, 662/apto11 - Vila Clementino - 04025-013 - São Paulo-SP - Fone: (011) 5576-4421 - Fax: 01155733371 -

e-mail: janineepm@uol.com.br / janine@denf.epm.br

Recebido em: 3/4/2006 Aceito com modificações em: 15/9/2006
}

Rev Bras Ginecol Obstet. 2006; 28(9): 523-9. 


\section{Introdução}

A pressão pode ser definida como a "habilidade de um músculo ou de um grupo muscular para desenvolver tensão que resulte em um esforço máximo, tanto dinâmico quanto estático em relação a uma resistência imposta" ${ }^{1}$. A pressão muscular perineal (PMP) pode ser avaliada por meio do perineômetro de Kegel, que utiliza como unidade de medida $\mathrm{mmHg}$, ou por um instrumento de retroalimentação digital com sensor vaginal inflável que mensura a pressão em $\mathrm{cm}$ de $\mathrm{H}_{2} \mathrm{O}^{1}$. Podese, ainda, usar a ultra-sonografia para detecção de atrofia muscular, bem como usar eletroestimulação como técnica de reeducação perineal, além do emprego do perineômetro de Kegel como método de biofeedback e de eletromiografia ${ }^{2}$.

A identificação da população de risco para a diminuição da PMP visa à profilaxia precoce para diminuir os agravos decorrentes como a insuficiência do assoalho pélvico, a incontinência urinária de esforço, a insuficiência anal e as disfunções sexuais $^{1}$. O processo reprodutivo nas mulheres exerce papel preponderante nas disfunções do assoalho pélvico, na maioria das vezes por causa das características anatômicas originais que facilitam o nascimento por via vaginal e, também, por causa do trauma que pode ocorrer durante esse evento ${ }^{3}$.

Identificam-se fatores intrínsecos relacionados à hereditariedade, que são obtidos por meio da história familiar com incontinência urinária, prolapso genital ou de tecido conjuntivo, e os fatores de risco extrínsecos, que são a paridade, a atividade profissional, os hábitos alimentares (em especial os que conduzem à obstipação), a bronquite crônica e a obesidade ${ }^{1}$.

O parto vaginal têm sido relacionado como um dos fatores precipitantes da incontinência urinária. Refere-se que a pressão do vértice fetal sobre o assoalho pélvico causa o progressivo estiramento e compressão dos nervos da junção e lesão da fáscia uretrovesical e do músculo elevador do ânus, podendo causar trauma neuromuscular ao períneo ${ }^{4}$.

Atualmente, um dos benefícios atribuídos ao parto cesáreo é reduzir o risco de aparecimento de problema comum no pós-parto, a incontinência urinária ${ }^{5}$.

Em estudo com primíparas, a incontinência urinária foi observada em $26 \%$ das mulheres, seis meses após o parto. Tiveram taxas mais baixas aquelas submetidas a parto cesáreo (5\%); quando eletivo após trabalho de parto, a taxa alcançou $12 \%$, elevando-se no nascimento por parto vaginal espontâneo $(22 \%)$ e, ainda mais, no vaginal por fórcipe $(33 \%)^{6}$.
O efeito traumático do parto vaginal é resultado de disfunção da musculatura do assoalho pélvico e esta disfunção deveria ser avaliada com mais atenção durante os exames ginecológicos de rotina. A musculatura pélvica compõe-se de fibras do tipo I (cerca de 70,0\%) e do tipo II (30,0\%), sendo as primeiras as responsáveis pela manutenção do tônus muscular, pela capacidade de contração lenta e de suportar longos períodos de solicitação sem sofrer fadiga. As fibras do tipo II são fatigáveis, realizam as contrações rápidas em resposta a aumentos súbitos de pressão intra-abdominal, o que acontece nas situações de tosse ou esforço repentino, porém não em trabalho de parto, uma vez que este constitui processo lento e gradual.

Há situações em que a passagem do feto pelo canal do parto ocasiona traumas que podem ser clinicamente identificados. Nestes casos classificam-se as lesões espontâneas decorrentes da passagem fetal pelas estruturas anatômicas do canal do parto em quatro graus. O primeiro grau envolve a fúrcula vaginal, a pele e/ou a mucosa vaginal. No segundo grau, atingem a musculatura e o corpo perineal, o terceiro estende-se ao esfincter anal e as de quarto grau, além do esfincter, ampliam-se até a mucosa retal, expondo o lúmen do reto ${ }^{7}$. A importância da classificação do grau das lesões está no fato da identificação precisa das estruturas envolvidas no agravo, permitindo a sutura adequada e reconstituição.

O manual da Organização Mundial de Saúde, traduzido no Brasil pelo Ministério da Saúde em 1996 sobre a "Maternidade Segura - Assistência ao Parto Normal: um guia prático", discorre sobre as recomendações de ações obstétricas para a redução das elevadas taxas de morbimortalidade nas mulheres em razão das rotinas indiscriminadas utilizadas no processo da parturição. Recomenda, como uma das estratégias para a diminuição dos riscos provocados pelos altos indices de parto cesáreo, o incentivo ao parto normal com o uso seletivo de episiotomia ${ }^{8}$.

A proteção perineal nem sempre é garantida pela incisão cirúrgica, em razão da dificuldade de recomposição da musculatura incisionada às condições prévias, pela própria dificuldade no reconhecimento adequado das estruturas a serem suturadas. Ao manter-se a integridade perineal, quando se assiste um parto eutócico sem complicações preserva-se o assoalho pélvico ${ }^{9}$.

A maioria dos estudos volta sua atenção às conseqüências do trauma perineal (incontinência urinária e anal, prolapso urogenital), mas há poucos estudos que focalizam a avaliação da musculatura do assoalho pélvico ${ }^{10}$.

Desta forma, nos propomos neste estudo a relacionar o tipo de parto e as características do perí- 
neo com valores da PMP mensurada em primíparas nas posições deitada e sentada com a musculatura perineal em repouso e em contração máxima.

\section{Métodos}

Estudo transversal no qual se avaliou a pressão muscular perineal em primíparas, assistidas em maternidade de baixo risco obstétrico conveniada ao Sistema Único de Saúde, no município de São Paulo. A casuística constitui-se de primiparas, sem doenças perineais prévias, que tiveram parto vaginal de feto único em apresentação cefálica, ou cesárea. Neste último, admitiu-se a apresentação pélvica por não ocorrer influência da distensão perineal do período expulsivo.

Durante 34 dias consecutivos foram oferecidas consultas de retorno no Ambulatório de Assistência a Puérperas a 692 mulheres internadas na unidade de pós-parto. Destas, 423 manifestaram interesse e tiveram a consulta puerperal de rotina agendada. Foram considerados como critérios de inclusão: ausência de doenças perineais por ocasião da admissão obstétrica, primigestas a termo (entre 37 e 42 semanas de gravidez), feto único e vivo em apresentação cefálica nos partos vaginais e, apresentação pélvica somente nos casos submetidos a cesárea, e, retorno para consulta de pós-parto entre o $40^{\circ}$ e o $45^{\circ}$ dia. Este período foi escolhido por permitir a avaliação da atividade sexual e a verificação de involução local em relação às condições pré-gravídicas.

Apesar do agendamento prévio das puérperas internadas, apenas 109 (25,8\%) compareceram à consulta. Houve exclusão de 14 usuárias, sendo seis por falha de agendamento, duas por retorno após o período estabelecido e seis por recusa ao exame ou participação na pesquisa.

Desta forma, a casuística, obtida por conveniência, totalizou 95 primíparas que retornaram à consulta puerperal tardia, e que apresentaram como características sociodemográficas: 60,0\% (57 casos) entre 20 e 32 anos; $36,8 \%$ (35) eram adolescentes, com idade entre 16 a 19 anos, e 3,2\% (3) tinham idade igual ou superior a 33 anos. Em relação à cor da pele, $52,6 \%$ (50) auto-referiram-se como não brancas e 47,4\% (45) como brancas. Quanto à situação marital, $75,8 \%$ (72) viviam com o companheiro, ao passo que $24,2 \%$ (23) não o tinham.

Considerou-se variável dependente e objeto do estudo a PMP, definida pela capacidade física da musculatura perineal em gerar tensão e deslocar determinada quantidade de massa por meio de contração ${ }^{11}$. Como instrumento de medida da PMP foi utilizado o perineômetro do tipo
Kegel, composto por um manômetro graduado entre 0 e $100 \mathrm{mmHg}$, calibrado, da marca B-D, $\mathrm{n}^{\circ} \mathrm{G} 93559$, conectado a uma pera ligeiramente cônica por uma extensão de látex com $80 \mathrm{~cm}$ de comprimento e mantidos em circuito fechado por meio de uma extensão de látex que registra a pressão muscular em $\mathrm{mmHg}^{12}$.

As variáveis independentes foram o tipo de parto (normal e cesárea), características do períneo (integro, com rotura espontânea e com episiotomia) e retorno à atividade sexual.

A cliente foi orientada em relação à retirada de roupas, esvaziamento prévio da bexiga, posição a ser assumida na mesa de exame e número de mensurações a serem obtidas. Possibilitouse o seu contato visual e tátil com o aparelho visando a familiarizá-la com o mesmo. Após o posicionamento da puérpera na posição ginecológica e com o aparelho preparado, foi realizada a introdução lenta do mesmo pelo intróito vaginal com um movimento de discreta inclinação para baixo até obter seu ajuste à cavidade vaginal, sendo exteriorizados apenas os $2 \mathrm{~cm}$ distais da pera de látex. A mão que introduziu esta pera manteve apoio externo para não haver expulsão do aparelho durante as contrações realizadas para as mensurações. A outra mão sustentou o manômetro na altura e proximidade do glúteo direito da puérpera. Após as três mensurações, na posição deitada, auxiliou-se a mulher a sentar-se na mesa ginecológica, tendo como apoio para os pés a escadinha frontal. A mão do examinador ficou levemente apoiada sobre a pera de látex para não ocorrer saída involuntária, durante o ato de sentar-se. Estando a puérpera sentada, realizaram-se as três leituras nesta posição. O manômetro foi mantido na mesma altura da pera e próximo à face interna da coxa direita. A pressão muscular em repouso foi mensurada pela massa deslocada com a musculatura em estado de repouso e a pressão de contração máxima foi mensurada quando se caracterizou a utilização de pressão contrátil máxima dos músculos perineais. As mensurações foram realizadas nas posições: deitada, em repouso e em contração máxima, e sentada, em repouso e em contração máxima, sendo aferidas três leituras em cada uma destas posições para extrair-se a média simples. Totalizaram-se 12 mensurações para cada puérpera examinada. O intervalo entre cada uma destas leituras foi 5 segundos, para permitir o relaxamento necessário às fibras musculares, e o tempo entre as duas posições (deitada/sentada) foi 15 segundos. Durante a execução do esforço máximo, solicitou-se sua manutenção durante cinco segundos e posterior relaxamen- 
to. Terminado o exame, foi retirada lentamente a pera, e ainda com a cliente sentada, foram retiradas as proteções-padrão do instrumento e da pesquisadora.

A coleta de dados ocorreu no período de 28 de abril a 31 de maio de 2002, após aprovação pela Diretoria Clínica da Maternidade e do Comitê de Ética da Universidade Federal de São Paulo. Houve esclarecimento prévio para todas as mulheres envolvidas e assinatura do termo de consentimento livre e esclarecido, em consonância com a Resolução no $196 / 96^{13}$.

Para as variáveis-tipo e características do parto foram aplicados os testes de KruskalWallis. Destinaram-se às comparações estatísticas das diferenças de intensidade da PMP, conforme o padrão de pressão analisado (deitada repouso/contração máxima e sentada repouso/ contração máxima). Para a análise estatística foi utilizado o programa SPSS - Social Package Statistical Science, versão 8.0 (1997), sendo fixado nivel de significância $\mathrm{p}<0,05$.

\section{Resultados}

Em relação ao tipo de parto, 23,2\% (22) das mulheres foram submetidas à cesárea e $76,8 \%$ (73) tiveram parto vaginal. No pós-parto vaginal verificaram-se as condições do períneo: $33,7 \%$ (32) tiveram episiotomia, em 24,2\% (23) houve rotura perineal (considerando-se todos os graus agrupados) e 18,9\% (18) tiveram a integridade perineal preservada (Figura 1).

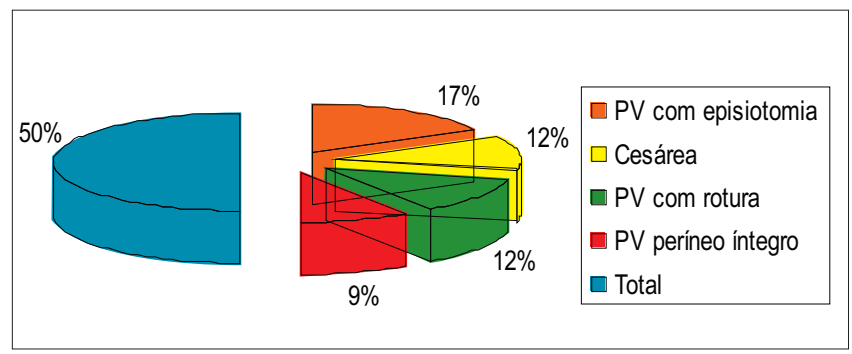

Figura 1 - Puérperas primíparas submetidas à mensuração da pressão muscular perineal, segundo o tipo de parto e as condições perineais.
Os valores obtidos pela perineometria relacionados ao tipo e características do períneo encontramse descritos na Tabela 1 . As mulheres submetidas ao parto cesárea tiveram valores médios da PMP 21,6; 34,$1 ; 37,5$ e $51,2 \mathrm{mmHg}$ maiores do que aquelas com episiotomia, rotura perineal e integridade perineal (PMP em mmHg:). O teste de Kruskal-Wallis demonstrou que, embora nas diferentes posições e estados da musculatura perineal o valor da PMP entre as mulheres submetidas ao parto cesáreo estivesse mais elevado, a diferença não se revelou significativa $(\mathrm{p}=$ 0,$12 ; 0,08 ; 0,29$ e 0,13).

Com a mulher na posição deitada e com a musculatura perineal em esforço, o maior valor da pressão muscular perineal ocorreu entre as mulheres que tiveram parto vaginal e cujos períneos mantiveram-se integros, seguidas daquelas com rotura espontânea e, por último, daquelas com partos com episiotomia.

A mensuração da PMP com a mulher sentada em repouso permitiu observar que, entre as submetidas ao parto vaginal com episiotomia, houve maior pressão, seguidas pelas de parto com integridade perineal preservada e pelas que tiveram rotura. $\mathrm{Na}$ posição sentada em esforço, nas mulheres que tiveram parto vaginal com períneo íntegro o valor da pressão foi maior do que nas submetidas a episiotomia, seguidas pelas que tiveram rotura. Mais da metade das puérperas, 54,7\% (52), ainda não haviam retornado à atividade sexual por ocasião da consulta de retorno puerperal, ao passo que 45,3\% (43) já haviam tido relação sexual, sendo que o valor médio da pressão muscular perineal nas quatro situações foi maior naquelas mulheres que não haviam retornado à atividade sexual até o momento da consulta. No entanto, com aplicação do teste de Mann-Whitney, somente na posição deitada em repouso observou-se diferença com significância estatística $(p<0,01)$.

Entre as mulheres que já haviam tido relação sexual antes da consulta de retorno, 62,8\% (27) consideraram-no prazeroso, ao passo que $34,9 \%$ (15) relataram dor e 2,3\% (1) tiveram a sensação de alteração vaginal, referindo como se sua vagina estivesse "mais larga" do que antes do parto.

Tabela 1 - Relação entre a pressão muscular perineal após o parto, as condições perineais e o tipo de parto.

\begin{tabular}{lcccccccc}
\hline & \multicolumn{8}{c}{ Pressão muscular perineal (mmHg) } \\
\cline { 2 - 9 } Tipo de parto e condições perineais & \multicolumn{1}{c}{ Deitada/Repouso } & \multicolumn{2}{c}{ Deitada/Esforço } & \multicolumn{2}{c}{ Sentada/Repouso } & \multicolumn{2}{c}{ Sentada/Esforço } \\
& Média & EPM $^{*}$ & Média & EPM & Média & EPM & Média & EPM \\
\hline Parto cesáreo & 21,6 & 1,6 & 34,1 & 1,6 & 37,5 & 2,1 & 51,2 & 2,4 \\
Parto vaginal com períneo íntegro & 16,9 & 1,2 & 31,7 & 2,4 & 33,2 & 1,9 & 46,2 & 2,6 \\
Parto vaginal com rotura & 19,1 & 1,4 & 29,5 & 2,2 & 33,0 & 2,2 & 42,7 & 2,8 \\
Parto vaginal com episiotomia & 18,1 & 1,2 & 28,8 & 1,7 & 34,3 & 1,7 & 46,0 & 2,2 \\
\hline
\end{tabular}

*EPM: Erro padrão da média; Teste de Kruskal-Wallis; Deitada/Repouso $p=0,125$; Deitada/Esforço $p=0,088$; Sentada/Repouso $p=0,298$; Sentada/Esforço $p=0,136$ 


\section{Discussão}

A taxa de cesárea entre as 95 primíparas que participaram do estudo foi $23,2 \%$. Entretanto, o indice geral desse indicador na maternidade neste período foi $16,7 \%$. Este indice de parto operatório está de acordo com o nível máximo de 15,0\% estabelecido pela Organização Mundial da Saúde (OMS) e consoante com a recomendação do Ministério da Saúde do Brasil, que preconiza taxas em torno de 15 a 20,0\%, considerando-se o total da população ${ }^{14}$.

No município de São Paulo, em 1998, esta taxa encontrava-se em torno de $35,0 \%$ entre as mulheres sem nenhuma escolaridade, e em torno de $73,0 \%$ nas de nivel superior, isto é, aquelas com maior poder de negociação com os serviços de saúde ${ }^{14}$. Estudo realizado utilizando perineometria, semelhante à metodologia aqui desenhada, obteve taxa de retorno de $15,2 \%$ pós-parto cesáreo e $84,8 \%$ pós-parto vaginal, em uma população de 105 puérperas. Ressalta-se que um dos objetivos do estudo era avaliar por meio do exame transvaginal a associação entre tipo de parto e lesão do nervo pudendo, além de contar com dois momentos de realização do exame, um na gestação acima de três semanas e outro entre a $6^{a}$ e a $8^{a}$ semana puerperal ${ }^{15}$.

Em estudo prospectivo com 128 primíparas com o objetivo de estabelecer o efeito do parto sobre as funções do nervo pudendo e identificar os fatores obstétricos associados com tais danos, avaliados durante a gravidez e de seis a oito semanas após o parto, mostrou-se que o parto vaginal provoca estiramento significativo do tecido do assoalho pélvico e danos ao nervo pudendo. As mulheres submetidas à cesárea durante o trabalho podem, também, ter risco destes danos. Os processos de trabalho de parto e parto vaginal provocam danos no nervo pudendo, que podem ter extensão assimétrica ${ }^{16}$.

$\mathrm{Na}$ literatura, não é citada associação entre PMP e tipo de parto. Apenas um estudo epidemiológico relacionando parto vaginal e incontinência urinária foi encontrado. Este estudo, com moradoras do condado de Høgskolen i Nord-Trøndelag
- Noruega (Epincont Study), envolveu 15.307 mulheres, divididas em três grupos: mulheres que não tiveram nenhum parto, que tiveram partos cesáreos e submetidas somente a parto vaginal; concluiu-se que o tipo de parto é fator de risco para este agravo à saúde. A prevalência da incontinência urinária foi 10,1\% nas nuliparas, 15,9\% nas submetidas a cesáreas e $21,0 \%$ nas que haviam tido parto vaginal ${ }^{17}$.

Existem poucos textos relacionando a estrutura do assoalho pélvico e a mensuração da PMP. Em geral, os trabalhos relacionam a mensuração desta pressão com a eficácia de técnicas terapêuticas empregadas em grupos de mulheres, freqüentemente incontinentes, no climatério ou na menopausa ${ }^{18}$.

Estudo clínico com 268 primigestas atendidas na clínica pré-natal com aproximadamente 20 semanas de gestação, randomizadas para testar o efeito de exercícios supervisionados do assoalho pélvico na incidência de incontinência de esforço pós-parto em primigestas de risco com mobilidade do colo vesical, comprovada por ultra-som, mostrou que não houve mudanças na mobilidade do colo vesical nem diferença na força do assoalho pélvico entre os grupos (controle e experimental) após os exercícios, embora todas aquelas que desenvolveram incontinência urinária pós-parto tiveram escores significantemente piores à perineometria em relação às continentes ${ }^{19}$.

Em estudo norueguês ${ }^{20}$, verificou-se que a freqüência de incontinência urinária é mais alta entre mulheres com cesárea do que em nuliparas, e ainda maior entre as mulheres com parto vaginal. No entanto, esta afirmação não deve ser utilizada para justificar aumento nas taxas de cesárea, pois o estudo não considerou a morbimortalidade e os custos gerados pela realização indiscriminada de cesarianas.

Numa amostra de 697 mulheres que tiveram partos vaginais, 356 primiparas e 341 multiparas, que mantiveram a integridade perineal ou sofreram roturas espontâneas de $1^{\circ}$ ou $2^{\circ}$ graus (com necessidade ou não de rafia), a atividade sexual foi satisfatória após 6 semanas de pós-parto. As

Tabela 2 - Relação entre a pressão muscular perineal e o retorno à atividade sexual, no pós-parto tardio.

\begin{tabular}{lccccccccc}
\hline \multirow{3}{*}{ Retorno à atividade sexual } & \multicolumn{8}{c}{ Pressão muscular perineal (mmHg) } \\
\cline { 2 - 10 } & \multicolumn{2}{c}{ Deitada/Repouso } & \multicolumn{2}{c}{ Deitada/Esforço } & \multicolumn{2}{c}{ Sentada/Repouso } & \multicolumn{2}{c}{ Sentada/Esforço } \\
& Média & EPM & Média & EPM & Média & EPM & Média & EPM \\
\hline Sim & 16,7 & 0,8 & 28,7 & 1,4 & 33,3 & 1,7 & 45,6 & 2,2 \\
Não & 20,7 & 1,0 & 32,4 & 1,3 & 35,5 & 1,1 & 47,2 & 1,5 \\
\hline
\end{tabular}

*EPM: Erro padrão da média; Teste de Mann-Whitney; PMP Deitada/Repouso $p<0,01$; PMP Deitada/Esforço $p=0,072 ;$ PMP Sentada/Repouso $p=0,279 ;$ PMP Sentada/Esforço $p=0,347$ 
mulheres submetidas a episiotomia ou que tiveram roturas espontâneas de $3^{\circ}$ ou $4^{\circ}$ graus referiram insatisfação no retorno sexual, desconforto, dor ou receio ${ }^{21}$.

Em estudo sobre o treinamento da musculatura do assoalho pélvico como tratamento de mulheres com incontinência urinária foi avaliado o efeito do trabalho de parto e parto com disfunções urinárias, intestinais e sexuais e conclui-se que há necessidade de estudos epidemiológicos populacionais, com metodologia apropriada ${ }^{22}$.

Estudo clínico de corte transversal, para avaliar a pressão muscular do assoalho pélvico (AP) pelo teste da avaliação da pressão do assoalho pélvico (AFA) e pelo uso do perineômetro em primíparas realizado no Hospital e Maternidade de Assis, São Paulo, com 94 mulheres, permitiu concluir que: o parto vaginal diminuiu a pressão muscular do AP de primíparas, avaliada pela AFA e pelo perineômetro, após 4 a 6 meses do parto; o parto vaginal em primíparas aumentou em 2,58 ou 2,31 vezes o risco relativo de diminuição da pressão muscular do AP, após 4 a 6 meses do

\section{Referências}

1. Amaro JL, Gameiro MO, Kawano PR, Padovani CR. Intravaginal electrical stimulation: a randomized, double-blind study on the treatment of mixed urinary incontinence. Acta Obstet Gynecol Scand. 2006;85(5):619-22.

2. Mayer R, Wells TJ, Brink CA, Clark P. Correlations between dynamic urethral profilometry and perivaginal pelvic muscle activity. Neurourol Urodyn. 1994;13(3):227-35.

3. Patel DA, Xu X, Thomason AD, Ransom SB, Ivy JS, DeLancey JO. Childbirth and pelvic floor dysfunction: an epidemiologic approach to the assessment of prevention opportunities at delivery. Am J Obstet Gynecol. 2006;195(1):23-8.

4. Retzky SS, Rogers RM Jr. Urinary incontinence in women. Clin Symp. 1995;47(3):2-32.

5. Hannah ME. Planned elective cesarean section: a reasonable choice for some women? CMAJ. 2004;170(5):813-4. parto; o parto cesáreo em primíparas também aumentou em 1,56 ou 1,37 vezes o risco relativo de diminuição da pressão muscular do AP, após 4 a 6 meses do parto, porém o IC a 95\% não foi significativo ${ }^{23}$.

Os autores, ainda, preconizam a necessidade de novas investigações importantes para o nosso país, onde são elevadas as taxas de cesárea. É preciso definir, em longo prazo, se realmente essas taxas são abusivas ou se estão corretas e há beneficio para as mulheres.

A análise e discussão dos resultados permitem concluir: a comparação entre os valores da PMP mensurada, nas quatro posições no pós-parto de puérperas primíparas, não apresentou relação com o tipo de parto e as condições perineais.

As principais implicações para a prática obstétrica estão relacionadas a rever o papel do parto cesáreo para minimizar a morbidade neurofisiológica resultante do trabalho de parto e do parto vaginal. As gestantes de alto risco poderiam escolher esta modalidade de parto, especialmente quando a intervenção adotada for o fórcipe.

6. Farrell SA, Allen VM, Baskett TF. Parturition and urinary incontinence in primiparas. Obstet Gynecol. 2001;97(3):350-6.

7. Cunningham FG, MacDonald PC, Gant NF, Leveno KJ, Gilstrap LC, Hankins GDV, et al. Williams Obstetrícia. 20a ed. Rio de Janeiro: Guanabara Koogan; 2000. p. 294-9.

8. Organização Mundial da Saúde. Maternidade segura. Assistência ao parto normal: um guia prático. Relatório de um grupo técnico. Genebra: OMS; 1996. (OMS/SRF/MSM/96.24).

9. Myers-Helfgott MG, Helfgott AW. Routine use of episiotomy in modern obstetrics. Should it be performed? Obstet Gynecol Clin North Am. 1999;26(2):305-25

10.Sartore A, De Seta F, Maso G, Pregazzi R, Grimaldi E, Guaschino S. The effects of mediolateral episiotomy on pelvic floor function after vaginal delivery. Obstet Gynecol. 2004;103(4):669-73. 
11.Santarém JM. Treinamento de pressão e potência. In: Gorayeb N, Barros Neto TL, editores. O exercício: preparação fisiológica, avaliação médica, aspectos especiais e preventivos. São Paulo: Atheneu; 1999. p. 35-7.

12.Kegel AH. Progressive resistance exercise in the functional restoration of the perineal muscles. Am J Obstet Gynecol. 1948;56 (1):238-48.

13. Conselho Nacional de Saúde [homepage da Internet]. Resoluçao $\mathrm{n}^{\circ} 196$, de 10 de outubro de 1996. Diretrizes sobre pesquisas que envolvem seres humanos [citado 2006 Ago 3]. Disponivel em: http: / /www.unifesp.br/reitoria/orgaos/comites / etica/Reso196.doc

14. Freitas PF, Drachler ML, Leite JCC, Grassi PR. Desigualdade social nas taxas de cesariana em primíparas no Rio Grande do Sul. Rev Saúde Pública. 2005;39(5):761-7.

15. Ministério da Saúde. Parto, aborto e puerpério. Assistência humanizada à mulher. Brasília: Ministério da Saúde; 2001. p. 38-44.

16.Sultan AH, Kamm MA, Hudson CN. Pudendal nerve damage during labour: prospective study before and after childbirth. Br J Obstet Gynaecol. 1994;101(1):22-8.

17.Rortveit G, Daltveit AK, Hannestad YS, Hunskaar S; Norwegian EPINCONT Study. Urinary incontinence after vaginal delivery or cesarean section. N Engl J Med. 2003;348(10):900-7.

18.Pregazzi R, Sartore A, Bortoli P, Grimaldi E, Ricci $\mathrm{G}$, Guaschino S. Immediate postpartum perineal examination as a predictor of puerperal pelvic floor dysfunction. Obstet Gynecol. 2002;99(4):581-4.

19.Glashan RQ, Lelis MAS, Fera P, Bruschini H. Intervenções comportamentais e exercícios perineais no manejo da incontinência urinária em mulheres idosas. Sinopse Urol. 2002;6(5):102-6.

20.Chung SY, Franks M, Smith CP, Lee JY, Lu SH, Chancellor M. Technique of combined pubovaginal sling and cystocele repair using a single piece of cadaveric dermal graft. Urology. 2002;59(4):538-41.

21.Klein MC, Gauthier RJ, Robbins JM, Kaczorowski J, Jorgensen SH, Franco ED, et al. Relationship of episiotomy to perineal trauma and morbidity, sexual dysfunction, and pelvic floor relaxation. Am J Obstet Gynecol. 1994;171(3):591-8.

22.Hay-Smith EJ, Dumoulin C. Pelvic floor muscle training versus no treatment, or inactive control treatments, for urinary incontinence in women. Cochrane Database Syst Rev. 2006;(1):CD005654

23. Barbosa AMP, Carvalho LR, Martins AMVC, Calderon IMP, Rudge MVC. Efeito da via de parto sobre a força muscular do assoalho pélvico. Rev Bras Ginecol Obstet. 2005;27(11):677-82. 